\title{
Asymptotics of the Solution of an Integral Equation to Transmission Problems with Singular Perturbed Boundary
}

\author{
R. Mahnke
}

Abstract. The integral equation to a transmission problem of the Laplacian is considered on a smooth boundary of a plane domain. The contour depends on a positive parameter $\varepsilon$ and the domain has a corner in the limit case $\varepsilon=0$. The main terms of an asymptotic expansion showing the influence of the parameter are given. The remaining part is estimated in a weak norm.

Keywords: Asymptotics, boundary integral equations, transmission problems

AMS subject classification: $35 \mathrm{~J} \mathrm{05,} 45 \mathrm{M} \mathrm{05,} 31 \mathrm{~B} \mathrm{10,35 \textrm {B }} 40$

\section{Introduction}

A large number of investigations have been devoted to elliptic boundary value problems in domains with conical points. The asymptotic behaviour of solutions in a neighbourhood of the singular points is well-known (see Kondratyev [1], and Maz'ya and Plamenevsky [6, 7]). In [5] Maz'ya, Nazarov and Plamenevsky developed a method which demonstrates the influence of a small perturbation of the boundary near a singular point.

Let $\Omega$ be a plane domain which coincides with an angle in a neighbourhood of the origin and let $\Omega_{\varepsilon}$ be a domain which is obtained by smoothing the corner of $\Omega$. Then the solution of an elliptic boundary value problem

$$
\left.\begin{array}{ll}
L u_{e}=f & \text { in } \Omega_{\varepsilon} \\
B u_{\varepsilon}=g & \text { on } \partial \Omega_{\varepsilon}
\end{array}\right\}
$$

has the representation

$$
u_{e}(x)=\sum_{k=0}^{+\infty}\left(\varepsilon^{\sigma_{k}} v_{k}(x)+\varepsilon^{r_{k}} w_{k}\left(\frac{x}{\varepsilon}\right)\right) .
$$

The parameter $\varepsilon$ is a size of the perturbation of the corner. The sequences $\left\{\sigma_{k}\right\}_{k \in N_{0}}$ and $\left\{\tau_{k}\right\}_{k \in N_{0}}$ of real numbers are monotonously increasing. The functions $v_{k}\left(k \in \mathbb{N}_{0}\right)$

R. Mahnke: University of Rostock, Department of Mathematics, D - 18051 Rostock 
are solutions of boundary value problems with respect to $\Omega$, whereas the functions $w_{k}\left(k \in \mathbb{N}_{0}\right)$ solve boundary value problems in an unbounded domain $\omega$ which does not depend on $\varepsilon$ too and is obtained by a transformation of coordinates $\xi=\frac{x}{\varepsilon}$.

It is possible to apply.this method even to solutions of boundary integral equations as was shown in [4], where the main terms of the series were given for the integral equation to the Dirichlet problem of the Laplacian. The occuring functions were restrictions of solutions of boundary value problems to the boundary, but not solutions of an integral equation itself.

In the present paper we investigate the solution $\mu_{\varepsilon}$ of the boundary integral equation

$$
\left(\frac{1}{2} I-\lambda K^{*}\right) \mu_{\varepsilon}=f_{\varepsilon}
$$

on the boundary $\partial \Omega_{\varepsilon}$ of the domain $\Omega_{e}$, where $I$ denotes the identical operator, $\lambda \in(0,1)$ is a real number and $K$ is the direct value of the double layer potential

$$
K \mu_{\varepsilon}(x)=\frac{1}{2 \pi} \int_{\partial \Omega_{\varepsilon}} \mu_{\varepsilon}(y) \frac{\partial}{\partial \nu_{y}}(\ln |x-y|) d s_{y}
$$

with normal $\nu$ directed outward. The adjoint operator $K^{*}$ is the direct value of the normal derivative of the simple layer potential

$$
S \mu_{\varepsilon}(x)=\frac{1}{2 \pi} \int_{\partial \Omega_{\varepsilon}} \mu_{\varepsilon}(y) \ln |x-y| d s_{y}
$$

on $\partial \Omega_{\epsilon}$.

We will derive the following representation for $\mu_{\varepsilon}$ on $\partial \Omega_{\varepsilon}$ :

$$
\mu_{\varepsilon}(x)=\mu(x)+\varepsilon^{\tau_{0}-1} \rho\left(\frac{x}{\varepsilon}\right)+R(x)
$$

where $\mu$ and $\rho$ can be considered as solutions of boundary integral equations which are independend on $\varepsilon$. The real number $\tau_{0}$ is the smallest positive eigenvalue of a transmission problem with respect to the corresponding angle. An estimate of the remainder function $R=R(x)$ is given in the $L_{2}$-norm.

\section{A transmission problem}

Let $B_{1}(0)$ denote the unit circle with center in the origin $O$ and let $\Omega \subset \mathbb{R}^{2}$ be a bounded domain which coincides with the angle

$$
G=\{x=(r, \varphi) \mid r>0 \text { and } \varphi \in(0, \alpha)\} \quad(0<\alpha<2 \pi)
$$

inside $B_{1}(O)$, where $r$ and $\varphi$ denote polar coordinates. We assume that $\partial \Omega \backslash\{O\}$ is smooth. Let a second domain $\omega \subset \mathbb{R}^{2}$ have smooth boundary and let it coincides with 
$G$ outside $B_{1}(O)$. For sake of simplicity, we assume $\Omega \subset G$ and $\omega \subset G$. We obtain corresponding domains $\omega_{\varepsilon}, \Omega_{\varepsilon}$ and $\widetilde{\Omega}_{\varepsilon}$ introducing a parameter $\varepsilon(1>\varepsilon>0)$ :

$$
\omega_{e}=\left\{x \mid \frac{x}{e} \in \omega\right\}, \quad \Omega_{e}=\Omega \cap \omega_{e}, \quad \widetilde{\Omega}_{e}=\left\{\xi \mid \xi \varepsilon \in \Omega_{e}\right\}
$$

We consider the following transmission problem with respect to $\Omega_{\varepsilon}$, which is known as electrostatic problem (see [2]):

$$
\begin{aligned}
\Delta u_{\varepsilon} & =0 & & \text { in } \mathbb{R}^{2} \backslash \partial \Omega_{e} \\
u_{e}^{+}-u_{e}^{-} & =0 & & \text { on } \partial \Omega_{e} \\
(1-\lambda) \frac{\partial u_{e}^{+}}{\partial \nu}-(1+\lambda) \frac{\partial u_{e}}{\partial \nu} & =\frac{\partial V_{0}}{\partial \nu} & & \text { on } \partial \Omega_{\varepsilon} \\
u_{\varepsilon}(x) & =o(1) & & \text { for }|x| \rightarrow \infty
\end{aligned}
$$

where $V_{0}$ is a given potential harmonic in the whole plane, the superscripts + and - indicate the limits at the boundary $\partial \Omega_{\varepsilon}$ from outside and inside, respectively, and $\lambda \in(0,1)$.

Seeking the solution $u_{\varepsilon}$ of problem (1) in form of a single layer potential $u_{\varepsilon}=S \mu_{\varepsilon}$, the well-known jump conditions (see, e.g., [3]) yield the integral equation

$$
\frac{1}{2} \mu_{e}-\lambda K^{*} \mu_{\varepsilon}=\frac{1}{2} \frac{\partial V_{0}}{\partial \nu}=: f_{e}
$$

Additionally, the jump conditions lead to

$$
\mu_{\varepsilon}(x)={\frac{\partial u_{\varepsilon}}{\partial \nu}}^{+}-\frac{\partial u_{\varepsilon}{ }^{-}}{\partial \nu}=\frac{1}{1-\lambda}\left(f_{\varepsilon}+2 \lambda{\frac{\partial u_{\varepsilon}}{\partial \nu}}^{-}\right)
$$

Therefore, the desired representation of $\mu_{\varepsilon}$ in form of a series can be found by applying the method of Maz'ya, Nazarov and Plamenevsky to the function $u_{\varepsilon}$. Doing so we get formal asymptotics

$$
u_{\varepsilon}(x)=v_{0}(x)+\varepsilon^{\tau_{0}} w_{0}\left(\frac{x}{\varepsilon}\right)+R_{1}(x) .
$$

The remainder function $R_{1}=R_{1}(x)$ will be considered in section 4 . The function $v_{0}$ solves the following problem with respect to $\Omega$, which corresponds to problem (1):

$$
\begin{aligned}
\Delta v_{0} & =0 & & \text { in } \mathbb{R}^{2} \backslash \partial \Omega \\
v_{0}^{+}-v_{0}^{-} & =0 & & \text { on } \partial \Omega \\
(1-\lambda){\frac{\partial v_{0}}{\partial \nu}}^{+}-(1+\lambda){\frac{\partial v_{0}}{\partial \nu}}^{-} & =\frac{\partial V_{0}}{\partial \nu} & & \text { on } \partial \Omega \\
v_{0}(x) & =o(1) & & \text { for }|x| \rightarrow \infty .
\end{aligned}
$$


The domains in (1) and (5) differ only in a neighbourhood of the origin, where an essential difference between the solutions $u_{e}$ and $v_{0}$ can be expected. The asymptotic behaviour of $v_{0}$ near $O$ is well-known (see [8]):

$$
v_{0}(x)=v_{0}(O)+r^{\tau_{0}} a(\varphi)+\mathcal{O}(r)
$$

where $\tau_{0} \in\left(\frac{1}{2}, 1\right]$ is the smallest positive eigenvalue of the corresponding model problem for the angle $G$ and solves the equation

$$
\sin ^{2}(\pi \tau)=\lambda^{2} \sin ^{2}((\pi-\alpha) \tau)
$$

We find the function $w_{0}$ in the following manner:

The difference $u_{e}-v_{0}$ solves a certain transmission problem with respect to $\Omega_{\varepsilon}$. In order to get an approximation for this difference, we substitute $v_{0}$ by the main term of its asymptotics, expand the domain to $\omega_{\varepsilon}$ and carry out the transformation $\xi=\frac{x}{\varepsilon}$. Factoring out the power $\varepsilon^{\tau_{0}}$, we obtain a problem with respect to $\omega$. The solution $w_{0}$ of this problem does not depend on $\varepsilon$.

The question of unique solvability of the transmission problems mentioned above was handled in detail in [2]. The following proposition is valid for all these problems with small modifications.

Proposition 1. The integral equation (2) is uniquely solvable in $L_{2}\left(\partial \Omega_{\varepsilon}\right)$. The simple layer potential $S \mu_{e}$ is the unique solution of problem (1) in the space $L_{2}^{1}\left(\mathbb{R}^{2}\right)$ of functions with quadratically integrable generalized first derivatives and shows the behaviour $\mathcal{O}\left(|x|^{-1}\right)$ at infinity.

Proof. The right-hand side of equation (2) is sufficently smooth, since $V_{0}$ is harmonical. The operator $\frac{1}{2} I-\lambda K^{*}$ is invertible in $L_{2}$ even for Lipschitz boundary [9]. We integrate equation (2) over $\partial \Omega_{\varepsilon}$ and obtain $\int_{\partial \Omega_{\varepsilon}} \mu_{\varepsilon} d s=0$ taking into account that $K 1=\frac{1}{2}$ on $\partial \Omega_{\varepsilon}$. It follows immediately that $S \mu_{\varepsilon}$ shows the behaviour $\mathcal{O}\left(|x|^{-1}\right)$ at infinity. On the other hand we handle (1) as a variational problem. Considering the factor space $\dot{L}_{2}^{1}\left(\mathbb{R}^{2}\right)$ of $L_{2}^{1}\left(\mathbb{R}^{2}\right)$ with respect to constants, the lemma of Lax-Milgram can be applied which secures the unique solvability of problem (1) neglecting the condition at infinity. It is possible to choose the constant in a way that this condition is fulfilled, since $S_{\mu_{\varepsilon}}$ satisfies all demands of problem (1) and belongs to $L_{2}^{1}\left(\mathbb{R}^{2}\right)$

\section{Formal asymptotics of the solution of the integral equation}

Following the method described in the previous section, we derive asymptotics of $\mu_{\varepsilon}$. This is a formal result, since the behaviour of the remainder function still has to be investigated.

Theorem 1. The solution of the integral equation (2) has the following formal asymptotics on $\partial \Omega_{\varepsilon}$ :

$$
\mu_{\varepsilon}(x)=\mu(x)+\varepsilon^{T_{0}-1} \rho\left(\frac{x}{\varepsilon}\right)+R(x) .
$$


On $\partial \Omega_{\varepsilon} \cap \partial \Omega$ the function $\mu$ coincides with the solution $\tilde{\mu}$ of the integral equation

$$
\tilde{\mu}-2 \lambda K^{*} \tilde{\mu}=\frac{\partial V_{0}}{\partial \nu}
$$

which has to be solved on $\partial \Omega$.

On $\partial \Omega_{\varepsilon} \backslash \partial \Omega$ we have

$$
\mu=\frac{1}{1-\lambda}\left(\frac{\partial V_{0}}{\partial \nu}+2 \lambda \frac{\partial(S \tilde{\mu})}{\partial \nu}\right)
$$

where the simple layer potential operator $S$ integrates over $\partial \Omega$.

On $\partial \widetilde{\Omega}_{\varepsilon} \cap \partial \omega$ the function $\rho$ coincides with the solution $\tilde{\rho}$ of the integral equation

$$
\tilde{\rho}-2 \lambda K^{*} \tilde{\rho}=-\frac{4 \lambda^{2}}{1-\lambda} \frac{\partial}{\partial \nu}^{-} S\left(\frac{\partial\left(|\xi|^{\tau_{0}} a(\varphi)\right)}{\partial \nu}\right)
$$

which has to be solved on $\partial \omega$ and $S$ integrates over the boundary of $\Omega \backslash \bar{\omega}$. On $\partial \tilde{\Omega}_{\varepsilon} \backslash \partial \omega$ we have $\rho=\frac{\partial w}{\partial \nu}$ where $w$ is the solution of the Neumann problem

$$
\left.\begin{array}{ll}
\Delta w=0 & \text { in } \omega \\
\frac{\partial \omega}{\partial \nu}=\tilde{\rho} & \text { on } \quad \partial \omega .
\end{array}\right\}
$$

Proof. We set $R_{0}=u_{\varepsilon}-v_{0}$ and consider (1) and (5) as variational problems (see Proposition 1) with test functions $\phi \in \dot{L}_{2}^{1}\left(\mathbb{R}^{2}\right)$. Then $R_{0}$ satisfies the equation

$$
\begin{aligned}
& (1+\lambda) \int_{\Omega_{\varepsilon}} \nabla R_{0} \nabla \phi d x+(1-\lambda) \int_{\mathbb{R}^{2} \backslash \bar{\Omega}_{\varepsilon}} \nabla R_{0} \nabla \phi d x \\
& =2 \lambda \int_{\partial \Omega \backslash \partial \Omega_{e}}{\frac{\partial v_{0}}{\partial \nu}}^{-} \phi d s-2 \lambda \int_{\partial \Omega_{\varepsilon} \backslash \partial \Omega} \frac{\partial v_{0}}{\partial \nu} \phi d s+\int_{\Omega \backslash \bar{\Omega}_{\varepsilon}} \nabla V_{0} \nabla \phi d x .
\end{aligned}
$$

The problem is uniquely solvable (see [2]), since $v_{0} \in L_{2}^{1}\left(\mathbb{R}^{2}\right)$ and $V_{0}$ was assumed to be harmonic. The essential part of the right-hand side of (6) is given by the main term $r^{T_{0}} a(\varphi)$ of the asymptotics of $v_{0}$. As already mentioned in the previous section, it is compensated by the function $\varepsilon^{T_{0}} w_{0}\left(\frac{x}{\varepsilon}\right)$, where $w_{0}$ solves the variational problem

$$
\begin{aligned}
(1+\lambda) \int_{\omega} & \nabla w_{0} \nabla \phi d \xi+(1-\lambda) \int_{\mathbb{R}^{2} \backslash \bar{\omega}} \nabla w_{0} \nabla \phi d \xi \\
& =2 \lambda \int_{\partial G \backslash \partial \omega} \frac{\partial\left(|\xi|^{\tau_{0}} a(\varphi)\right)^{-}}{\partial \nu} \phi d s_{\xi}-2 \lambda \int_{\partial \omega \backslash \partial G} \frac{\partial\left(|\xi|^{\tau_{0}} a(\varphi)\right)}{\partial \nu} \phi d s_{\xi} .
\end{aligned}
$$


We denote the dirac-delta distribution by $\delta$ and define the following functions using polar coordinates:

$$
h_{1}(r)= \begin{cases}1 & \text { if }(r, \alpha) \in \partial G \backslash \partial \omega \\ 0 & \text { otherwise }\end{cases}
$$

and

$$
h_{2}(r)= \begin{cases}1 & \text { if }(r, 0) \in \partial G \backslash \partial \omega \\ 0 & \text { otherwise. }\end{cases}
$$

We set $h(\xi)=h_{1}(|\xi|) \delta(\varphi-\alpha)-h_{2}(|\xi|) \delta(\varphi-0)$ and

$$
g(\xi)= \begin{cases}\frac{\partial\left(|\xi|^{r_{0}} a(\varphi)\right)}{\partial \nu} & \text { on } \partial \omega \backslash \partial G \\ 0 & \text { on } \partial \omega \cap \partial G\end{cases}
$$

It follows

$$
\begin{array}{cl}
\Delta w_{0}= \begin{cases}0 & \text { in } \omega \\
-\frac{2 \lambda}{1-\lambda}|\xi|^{r_{0}-2} a^{\prime}(\varphi) h(\xi) & \text { in } \mathbb{R}^{2} \backslash \bar{\omega}\end{cases} \\
w_{0}^{+}-w_{0}^{-}=0 & \text { on } \partial \omega \\
(1-\lambda) \frac{\partial w_{0}}{\partial \nu}-(1+\lambda){\frac{\partial w_{0}}{\partial \nu}}^{-}=2 \lambda g & \text { on } \partial \omega \\
w_{0}(\xi)=o(1) & \text { for }|\xi| \rightarrow \infty
\end{array}
$$

Let $E(\xi, \eta):=\frac{1}{2 \pi} \ln |\xi-\eta|$. Using the Green formulae and the jump conditions of the simple- and double-layer potentials, we obtain the following equations for the limits of the normal derivatives of $w_{0}$ approaching the boundary $\partial \omega$ from inside and outside; respectively:

$$
\begin{aligned}
\frac{1}{2} \frac{\partial w_{0}}{\partial \nu} \overline{(\eta)}= & -\int_{\partial \omega} \frac{\partial w_{0}}{\partial \nu}(\xi) \frac{\partial E}{\partial \nu_{\eta}} d s_{\xi}+\frac{\partial}{\partial \nu} \int_{\partial \omega} w_{0}(\xi) \frac{\partial E}{\partial \nu_{\xi}} d s_{\xi} \\
\frac{1}{2}{\frac{\partial w_{0}}{\partial \nu}}^{+}(\eta)= & \int_{\partial \omega} \frac{\partial w_{0}}{\partial \nu}{ }^{+}(\xi) \frac{\partial E}{\partial \nu_{\eta}} d s_{\xi}-\partial \partial \nu^{+} \int_{\partial \omega} w_{0}(\xi) \frac{\partial E}{\partial \nu_{\xi}} d s_{\xi} \\
& +\frac{\partial}{\partial \nu}_{\mathbb{R}^{2} \backslash \bar{\omega}}^{+} \Delta w_{0}(\xi) E d \xi .
\end{aligned}
$$

We combine (10) with (8) and add equation (9). Taking into account the continuity of the normal derivative of the double-layer potential, we obtain

$$
\begin{aligned}
{\frac{\partial w_{0}}{\partial \nu}-2 \lambda K^{*}{\frac{\partial w_{0}}{\partial \nu}}^{-}=}^{-}-2 \lambda \int_{\partial \omega} g(\xi) \frac{\partial E}{\partial \nu_{\eta}} d s_{\xi}-\lambda g(\eta) \\
-2 \lambda \frac{\partial}{\partial \nu} \int_{\partial G \backslash \partial \omega} \frac{\partial\left(|\xi|^{\tau_{0}} a(\varphi)\right)}{\partial \nu} E d s_{\xi} .
\end{aligned}
$$


The outward normal at $\partial \omega \backslash \partial G$ becomes the inward normal with respect to the domain $G \backslash \omega$. Making use of the jump condition of the normal derivative of the simple-layer potential at $\partial \omega$, we can simplify the right-hand side:

$$
{\frac{\partial w_{0}}{\partial \nu}}^{-}-2 \lambda K^{*}{\frac{\partial w_{0}}{\partial \nu}}^{-}=-2 \lambda \frac{\partial}{\partial \nu}_{\partial(G \backslash \omega)}^{-} \frac{\partial\left(|\xi|^{\tau_{0}} a(\varphi)\right)}{\partial \nu} E d s_{\xi}
$$

We pass to the solution of the boundary integral equation by combining (4) and (3):

$$
\mu_{\varepsilon}(x)=\frac{1}{1-\lambda}\left(\frac{\partial V_{0}}{\partial \nu}+2 \lambda \frac{\partial v_{0}-}{\partial \nu}(x)\right)+\varepsilon^{r_{0}-1} \frac{2 \lambda}{1-\lambda} \frac{\partial w_{0}}{\partial \nu_{\xi}}\left(\frac{x}{\varepsilon}\right)+\frac{2 \lambda}{1-\lambda} \frac{\partial R_{1}-}{\partial \nu}(x) .
$$

With

$$
\mu(x)=\frac{1}{1-\lambda}\left(\frac{\partial V_{0}}{\partial \nu}+2 \lambda{\frac{\partial v_{0}}{\partial \nu}}^{-}\right), \quad \rho\left(\frac{x}{\varepsilon}\right)=\frac{2 \lambda}{1-\lambda}{\frac{\partial w_{0}}{\partial \nu_{\xi}}}^{-}, \quad R(x)=\frac{2 \lambda}{1-\lambda}{\frac{\partial R_{1}}{\partial \nu}}^{-}
$$

the theorem is proved

\section{Estimation of the remainder function}

In order to justify the formal asymtotics of the solution $\mu_{\varepsilon}$ of problem (1) given in Theorem 1, we will estimate the remainder function in the $L_{2}$-norm. Let $E(x, y)=$ $\frac{1}{2 \pi} \ln |x-y|$. The following lemma shows that the remainder function $R_{1}$ solves a certain integral equation.

Lemma 1. The interior limit of the normal derivative of the remainder function $R_{1}$ defined by equation (4) satisfies the following equation on $\partial \Omega_{\varepsilon}$ :

$$
\begin{array}{rl}
\frac{\partial R_{1}}{\partial \nu}-2 & 2 \lambda K^{*} \frac{\partial R_{1}}{\partial \nu} \\
=-\frac{\partial^{-}}{\partial \nu} \int_{\partial\left(G \backslash \omega_{\varepsilon}\right)} \frac{\partial f}{\partial \nu} E d s-2 \lambda \varepsilon^{\tau_{0}} \frac{\partial}{\partial \nu} \int_{\partial(G \backslash \Omega)}^{-} \frac{\partial}{\partial \nu_{x}} w_{0}\left(\frac{x}{\varepsilon}\right) E d s
\end{array}
$$

where $f$ is a function which shows the behaviour $\mathcal{O}\left(r^{\min \left\{r_{1}, 2\right\}}\right)$ near the origin and $\tau_{1}>1$ is the second positiv eigenvalue of the transmission problem with respect to angle $G$.

Proof. In the proof of Theorem 1 we considered the variational problem (6) which is solved by $R_{0}=u_{e}-v_{0}$. Combining this with (7), we obtain a variational problem for $R_{1}$ defined by $R_{1}(x)=R_{0}(x)-\varepsilon^{T_{0}} w\left(\frac{x}{e}\right)$. For sake of simplicity we introduce the notation

$$
f(x)=V_{0}(x)-V_{0}(0)+2 \lambda\left(v_{0}(x)-v_{0}(0)-r^{T_{0}} a(\varphi)\right) .
$$

The linear terms in the asymptotics of $2 \lambda v_{0}$ and $-V_{0}$ coincide. Consequently, the main term in the asymptotics of $f$ shows the behaviour $r^{\min \left\{r_{1}, 2\right\}}$ near the origin, where $\tau_{1}$ 
is the second positive eigenvalue of the transmission problem with respect to angle $G$. It. holds

$$
\begin{aligned}
& (1+\lambda) \int_{\Omega_{e}} \nabla R_{1} \nabla \phi d x+(1-\lambda) \int_{\mathbf{R}^{2} \backslash \overline{\Omega_{e}}} \nabla R_{1} \nabla \phi d x \\
& =\int_{\partial \Omega \backslash \partial \dot{\Omega}_{e}} \frac{\partial f^{-}}{\partial \nu} \phi d s-\int_{\partial \Omega_{e} \backslash \partial \Omega} \phi d s+2 \lambda \varepsilon^{\tau_{0}}\left(\int_{\partial G \backslash \partial \Omega} \frac{\partial w_{0}^{-}}{\partial \nu} \phi d s-\int_{\partial \Omega \backslash \partial G} \frac{\partial w_{0}}{\partial \nu} \phi d s\right)
\end{aligned}
$$

with test functions $\phi \in \dot{L}_{2}^{1}\left(\mathbb{R}^{2}\right)$. The desired integral equation is obtained by repeating the steps in the proof of Theorem 1, since the equations (9) and (10) are valid for the normal derivative of $R_{1}$ with respect to $\partial \Omega_{c}$

Theorem 2. The remainder function $R$ of Theorem 1 satisfies the estimate

$$
\|R\|_{L_{2}\left(\partial \Omega_{*}\right)} \leq C \varepsilon^{\kappa}
$$

with $\kappa=\min \left\{\tau_{1}-\frac{1}{2}, \frac{3}{2}, 2 \tau_{0}\right\}$. The constant $C$ depends on $\lambda$ and the domains $\Omega$ and $\omega$, but does not depend on $\varepsilon$.

Proof. The operator $I-2 \lambda K^{*}$ is continously invertible in $L_{2,0}\left(\partial \Omega_{\epsilon}\right)$, the space of quadratically integrable functions with mean value 0 . This is valid even for Lipschitz boundaries (see [9] or [2]).

Combining equation $R(x)=\frac{2 \lambda}{1-\lambda} \frac{\partial R_{1}}{\partial \nu}$ and Lemma 1, the function $R$ satisfies an integral equation of the form $\left(I-2 \lambda K^{*}\right) R=F$ and the estimate

$$
\|R\|_{L_{2}\left(\partial \Omega_{\varepsilon}\right)} \leq\left\|\left(I-2 \lambda K^{*}\right)^{-1}\right\|\|F\|_{L_{2}\left(\partial \Omega_{t}\right)} \leq c\|F\|_{L_{2}\left(\partial \Omega_{\varepsilon}\right)}
$$

holds, where

$$
c=\sup _{\varepsilon \in(0,1)}\left\|\left(I-2 \lambda K^{*}\right)^{-1}\right\| .
$$

The supremum exists, since the operator is bounded even in the limit case $\varepsilon=0$.

Let $\tau=\min \left\{\tau_{1}, 2\right\}$ and $C$ be a constant which does not depend on $\varepsilon$. This constant may differ in different estimates and is equipped with subscripts in a sequence of estimates. It is sufficient to give an estimate for the right-hand side of (11) in the $L_{2}$-norm because of (12).

By changing the sequence of integration and normal derivative in the first term, an additional expression $\frac{1}{2} \frac{\partial f}{\partial \nu}$ occurs on $\partial \Omega_{e} \backslash \partial G$, which is caused by the jump condition. This term can be neglected, since it does not change the estimate. Let

$$
F_{1}(y)=\int_{\partial\left(\Omega \backslash \Omega_{\star}\right)} \frac{\partial f}{\partial \nu}(x) \frac{(y-x) \nu_{y}}{|y-x|^{2}} d s_{x}
$$

The behaviour $\frac{\partial f}{\partial \nu}(x)=\mathcal{O}\left(r^{r-1}\right)$ near $O$ yields after the transformation $\xi=\frac{x}{e}$ and $\eta=\frac{y}{\varepsilon}$

$$
\left|F_{1}(y)\right| \leq \underset{\partial\left(\Omega \backslash \Omega_{\varepsilon}\right)}{C}|x|^{\tau-1} \frac{\left|(y-x) \nu_{y}\right|}{|y-x|^{2}} d s_{x}=C \varepsilon^{r-1} \int_{\partial(G \backslash \omega)}|\xi|^{r-1} \frac{\left|(\eta-\xi) \nu_{\eta}\right|}{|\eta-\xi|^{2}} d s_{\xi} .
$$


Considering the $L_{2}$-norm of $F_{1}$, we devide the boundary $\partial \omega$ into 3 parts which belong to $B_{\varepsilon}(0), \quad B_{1}(0) \backslash \overline{B_{\varepsilon}(0)}$ and $\mathbb{R}^{2} \backslash \overline{B_{1}(0)}$, respectively. Taking into account that the expression

$$
\int_{\partial(G \backslash \omega)}|\xi|^{r-1} \frac{\left|(\eta-\xi) \nu_{\eta}\right|}{|\eta-\xi|^{2}} d s_{\xi}
$$

shows the behaviour $\mathcal{O}\left(|\eta|^{-1}\right)$ at infinity, we obtain

$$
\begin{aligned}
\left\|F_{1}\right\|_{L_{2}\left(\partial \Omega_{e}\right)}^{2} \leq & C_{1} \varepsilon^{2 \tau-2} \int_{\partial \omega \cap B_{1}(0)}\left(\int_{\partial(G \backslash \omega)}|\xi|^{\tau-1} \frac{\left|(\eta-\xi) \nu_{\eta}\right|}{|\eta-\xi|^{2}} d s_{\xi}\right)^{2} \varepsilon d s_{\eta} \\
& +C_{2} \varepsilon^{2 \tau-2}\left(\int_{\varepsilon}^{1} \frac{\varepsilon^{2}}{r^{2}} d r+\int_{\partial \Omega_{\varepsilon} \backslash B_{1}(0)} \frac{\varepsilon^{2}}{|y|^{2}} d y\right) \\
\leq & C \varepsilon^{2 \tau-1}
\end{aligned}
$$

For the estimate of the second term of the right-hand side of (11) we can neglect again the additional term on $\partial \Omega \backslash G$ caused by the jump condition.

Let

$$
F_{2}(y)=\varepsilon^{\tau_{0}} \int_{\partial(G \backslash \Omega)} \frac{\partial w_{0}}{\partial \nu}\left(\frac{x}{\varepsilon}\right) \frac{(y-x) \nu_{y}}{|y-x|^{2}} d s_{x}
$$

The function $\frac{\partial w_{0}}{\partial \nu_{\xi}}(\xi)$ shows the behaviour $\mathcal{O}\left(|\xi|^{-\tau_{0}-1}\right)$ at infinity. Hence

$$
\begin{aligned}
\left\|F_{2}\right\|_{L_{2}\left(\partial \Omega_{\varepsilon}\right)}^{2} & =\varepsilon^{2 \tau_{0}} \int_{\partial \Omega_{\varepsilon}}\left(\int_{\partial(G \backslash \Omega)} \frac{\partial w_{0}}{\partial \nu}\left(\frac{x}{\epsilon}\right) \frac{(y-x) \nu_{y}}{|y-x|^{2}} d s_{x}\right)^{2} d s_{y} \\
& \leq C_{1} \varepsilon^{4 \tau_{0}} \int_{\partial \Omega_{a}}\left(\int_{\partial(G \backslash \Omega)}|x|^{-\tau_{0}-1} \frac{\left|(y-x) \nu_{y}\right|}{|y-x|^{2}} d s_{x}\right)^{2} d s_{y} \\
& \leq C \varepsilon^{4 \tau_{0}}
\end{aligned}
$$

since the integral is bounded independly on $\varepsilon$. The estimates (12) - (14) complete the proof of Theorem 2 


\section{References}

[1] Kondratyev, V. A.: Boundary value problems for elliptic equations in domains with conical or angular points. Trans. Mosc. Math. Soc. 16 (1967), $227-313$.

[2] Mahnke, R.: Regularität und Asymptotik der Lösungen elliptischer Transmissionsprobleme in Lipschitzgebieten. Dissertation. Uni. Rostock 1991.

[3] Maz'ya, V. G.: Linear and Boundary Integral Equations (Encyclopaedia of Mathematical Sciences: Vol. 27). Berlin: Springer-Verlag 1991.

[4] Maz'ya, V. G. and R. Mahnke: Asymptotics of the solution of a boundary integral equation under a small perturbation of a comer. Z. Anal. Anw. 11 (1992), 173 - 182.

[5] Maz'ya, V. G., Nazarov, S. A. and B. A. Plamenevsky: Asymptotische Theorie elliptischer Randwertaufgaben in singulär gestörten Gebieten, Vol. 1. Berlin: A kademie-Verlag 1991.

[6] Maz'ya, V. G. and B. A. Plamenevsky: Estimates in $L_{p}$ and in Hölder classes and the Miranda-Agmon maximum principle for solutions of elliptic boundary value problems in domains with singular points on the boundary. Amer. Math. Soc. Transl. (2) 123 (1984), $1-56$.

[7] Maz'ya, V. G. and B. A. Plamenevsky: On the coefficients in the asymptotics of solutions of elliptic boundary value problems in domains with conical points. Amer. Math. Soc. Transl. (2) 123 (1984), $57-88$.

[8] Nicaise, S.: Polygonal interface problems. Frankfurt/M.: Verlag Peter Lang 1993.

[9] Verchota, G., Escauriaza, L. and E. B. Fabes: On a regularity theorem for weak solutions to transmission problems with internal Lipschitz boundaries. Proc. Amer. Math. Soc. 115 (1992), 1069 - 1076.

Received 28.11.1995 